\title{
Investigação preliminar de variações térmicas na superfície corporal de equinos tratados com Reiki
}

\author{
Preliminary investigation of body surface temperature variation on horses treated with Reiki
}

Luciana Lordello , Márcia T. Ramos ${ }^{\dagger, \S}$, Andrei Costa ${ }^{\dagger}$, Maria Fernanda de Mello Costall, o

Como citar esse artigo. Lordello,

L; Ramos, MT; Costa, A; de Mello

Costa, MF. Investigação preliminar de variações térmicas na superfície corporal de equinos tratados com Reiki. Revista de Saúde. 2019 Jan../Jun.; 10 (1): 20-26.

\begin{abstract}
Resumo
O Reiki é uma terapia energética antiga, originada no século dezoito no Japão. Apesar de relatos não documentados do alívio de sintomas e melhoria na cicatrização de pacientes humanos e animais tratados com o Reiki, há pouca evidência científica que comprove sua eficácia, e menos ainda em relação ao seu modo de ação. Este trabalho investigou as alterações de temperatura de superfície antes, durante e após sessões de Reiki em 6 cavalos, com o auxílio de um termógrafo FLIR. Variações individuais, associadas ao ponto de mensuração e ao tempo em relação ao tratamento com Reiki foram identificadas. Este estudo preliminar possibilitou a validação da metodologia a ser utilizada em estudos de maior escala, além de auxiliar na identificação de possíveis fontes de variação na temperatura de superfície em equinos tratados com Reiki. Mudanças de temperatura de superfície mais evidentes possivelmente seriam detectadas em animais doentes, em oposição a animais saudáveis. Estudos com maior número de animais e com a inclusão de medidas de temperatura corporal interna e perfil hematológico são necessários para esclarecer os efeitos mensuráveis da terapia Reiki em cavalos.
\end{abstract}

Palavras-chave: cavalos, imagem termográfica, terapias alternativas.

\begin{abstract}
Reiki is an ancient energetic therapy originated in the 18th Century in Japan. Although there is anedoctal evidence that Reiki causes relief of symptoms and improved healing in human and animal patients, there is little scientific evidence to support its efficacy and even less information on its mode of action. This work investigated superficial temperature changes before, during and after Reiki sessions in 6 horses with the aid of a FLIR thermograph. Individual subject variations, as well as changes associated with the point of measurement and time during treatment were identified. This is a preliminary study were methodology for larger studies was validated, as well as helping identify sources of surface temperature variation on horses treated with Reiki. More evident changes in temperature associated with Reiki treatment might be captured in sick animals, as opposed to clinically healthy ones. Studies with larger number of animals and with addition of body internal temperature measurements and haematology profiling are needed to further clarify the measurable effects of Reiki therapy on horses.
\end{abstract}

Keywords: alternative therapies, horses, thermographic imaging.

\section{Introdução}

A medicina veterinária vem expandindo sua área de atuação e, muitas vezes, por ação dos tutores dos animais, a busca por terapias alternativas vem ganhando destaque como opção de tratamento ou como coadjuvante do tratamento convencional de distúrbios de comportamento e afecções crônicas. O uso da acupuntura $^{1,2}$, florais $^{3}$, fitoterapia ${ }^{4}$ e terapias energéticas ${ }^{5,6}$ já foram descritos em medicina veterinária.

O Reiki, tipo de terapia energética muito utilizado em humanos, vem ganhando destaque no tratamento de animais por ser não invasivo e não requerer, em algumas situações, a presença do terapeuta e do animal no mesmo espaço físico. Desenvolvido no Japão pelo
Mestre Mikao Usui ${ }^{7}$, a terapia Reiki é um método de recomposição energética complementar reconhecido em diversas culturas, possuindo inclusive associações reguladoras como a Associação Portuguesa de Reiki (www.associacaoportuguesadeReiki.com) e o Conselho de Reiki da Índia (http://Reikicouncilofindia.com). O Reiki utiliza a imposição de mãos e a canalização energética para que o paciente possa acelerar o retorno à homeostase, e embora o princípio exato seja incerto, acredita-se que a base esteja na biofísica ${ }^{8}$. Durante o tratamento, o terapeuta utiliza pontos vitais energéticos chamados em várias culturas de "chacras". Estes pontos estão localizados em vários locais do corpo, sendo os sete principais localizados ao longo da coluna dorsal, sobre pontos onde existem plexos nervosos e glândulas endócrinas 9 .

\footnotetext{
Afiliação dos autores:

* Discente do Curso de Graduação em Medicina Veterinária da Universidade de Vassouras, Vassouras, Brasil;

† Docente do Curso de Graduação em Medicina Veterinária da Universidade de Vassouras, Vassouras, Brasil;

$\S$ Doutoranda do Curso de Medicina Veterinária da UFRRJ, Seropédica, Brasil;

\| Researcher, Centaur Equine Research Consortium, Hamilton, Nova Zelândia;

${ }^{\circ}$ Research Associate, Waikato Institute of Technology, Hamilton, Nova Zelândia. 
Com a crescente procura por novos métodos diagnósticos e tratamentos na Medicina Veterinária, além da crescente quantidade de informação disponível aos guardiães/tutores de animais torna-se importante obter comprovações científicas que justifiquem a utilização de alguns métodos classificados no âmbito da medicina alternativa.

Existem poucos trabalhos científicos que investiguem objetivamente o efeito da terapia Reiki sobre pacientes, sobressaindo-se as revisões sistemáticas que indicam que não há conclusões definitivas quanto à sua eficácia, sendo necessários maiores estudos de casos-controle ou randomizados ${ }^{10,11}$. No entanto, existem evidências de que a terapia Reiki é capaz de causar alterações significativas na pressão diastólica e frequência cardíaca ${ }^{12}$ e nos valores de hemoglobina ${ }^{11}$ em comparação com grupos controle e/ou placebo. Existem também trabalhos documentando a eficácia do Reiki em reduzir a sensação dolorosa ${ }^{13} \mathrm{e}$ em ativar culturas celulares ${ }^{14}$.

Existem relatos não documentados sobre o uso do Reiki na medicina veterinária com bons resultados ${ }^{15,16}$, sendo indicado como terapia complementar a tratamentos convencionais, agregando valor à medicina veterinária e atendendo aos interesses dos tutores, criadores e veterinários por novas terapias. O Reiki só deve ser ministrado por terapeutas treinados e certificados, e em associação com terapias tradicionais.

Não há relatos do efeito da terapia Reiki sobre a temperatura de superfície em animais, comparando-se a mesma antes e após o tratamento. Como o Reiki visa a homeostase, pode-se hipotetizar que a temperatura aumente ou diminua, dependendo do estado inicial do paciente.

Este trabalho tem como objetivo investigar, através da mensuração de temperatura de superfície corporal por meio de termografia, possíveis variações térmicas antes, durante e após o tratamento de Reiki, realizado por terapeuta qualificada, durante seções terapêuticas solicitadas pelos proprietários dos animais.

Foi escolhida a termografia infravermelha como método para a mensuração da temperatura de superfície por ser um método de fácil aplicação e bastante validado na medicina veterinária ${ }^{17-19}$.

\section{Material e métodos}

Este estudo foi aprovado pelo CEUA sob o número 028/2018. Foram utilizados seis equinos, todos da mesma propriedade e submetidos às mesmas condições higiênico sanitárias e alimentares. Os animais, compreendendo machos e fêmeas, com idades entre dois e seis anos de idade, são atletas da raça PuroSangue Inglês utilizados em corridas. São mantidos sob esquema de treinamento seis dias por semana, sempre na parte da manhã, com média de uma competição ao mês. Os animais incluídos no estudo foram considerados clinicamente saudáveis, através de exame prévio (antes da coleta de dados) realizado por uma veterinária especialista em medicina equina.

Após o exame clínico, que não foi observado por nenhum dos demais participantes do estudo, a terapeuta de Reiki demarcou, com fita adesiva, os locais dos principais chacras (pontos vitais energéticos), em um total de sete pontos corporais, além de quatro pontos distais, nos quatro membros do animal, equivalentes à pontos chamados de "botões". As figuras 1 e 2 demonstram a demarcação dos pontos onde o Reiki foi aplicado e a mensuração da temperatura obtida.

Imediatamente antes do início do tratamento de Reiki (Antes), durante o tratamento, na fase inicial (após a abertura de cada chacra, D1), durante a fase final do tratamento (foco de energia, D2) e ao final do tratamento como um todo (Após), uma das pesquisadoras, sem qualquer treinamento de Reiki, realizou, em triplicata, a medida da temperatura superficial de cada ponto identificado com fita adesiva (num total de 132 mensurações). As medidas de temperatura de superfície foram realizadas com um termógrafo FLIR modelo TG165, como demonstra a Figura 3 e anotadas em planilha desenvolvida para este fim.

Os resultados das mensurações de temperatura em triplicata foram transformados em média, resultando em uma média por ponto, por momento, por animal. Os resultados obtidos foram planilhados e submetidos à análise de variância (ANOVA) com dois fatores, estabelecendo-se o nível de significância em 95\% (p $\leq 0,05$ ). Os resultados da estatística descritiva estão apresentados como média \pm desvio padrão (DP).

\section{Resultados}

Foi realizado o cálculo do coeficiente de variação intra-ensaio (CVIE) para o equipamento através da medida consecutiva de um mesmo ponto sobre um objeto inanimado. Foram realizadas 21 mensurações, obtendo-se um CVIE de 2,75\%.

O cálculo de variação inter-ensaio, para dias consecutivos não foi realizado tendo em vista que todas as mensurações do experimento foram realizadas em um mesmo dia.

O experimento foi realizado das 16 às 19 horas de um dia nublado, com temperatura média no horário de realização do experimento de $21,7{ }^{\circ} \mathrm{C}$ (mínima 21,4 ${ }^{\circ} \mathrm{C}$ e máxima $22,0{ }^{\circ} \mathrm{C}$ ) e umidade média de $88,9 \%$. No dia da coleta, da meia noite às 23 horas, a média de temperatura foi de $21,9^{\circ} \mathrm{C}^{20}$.

Os pontos de mensuração sobre o corpo e os 

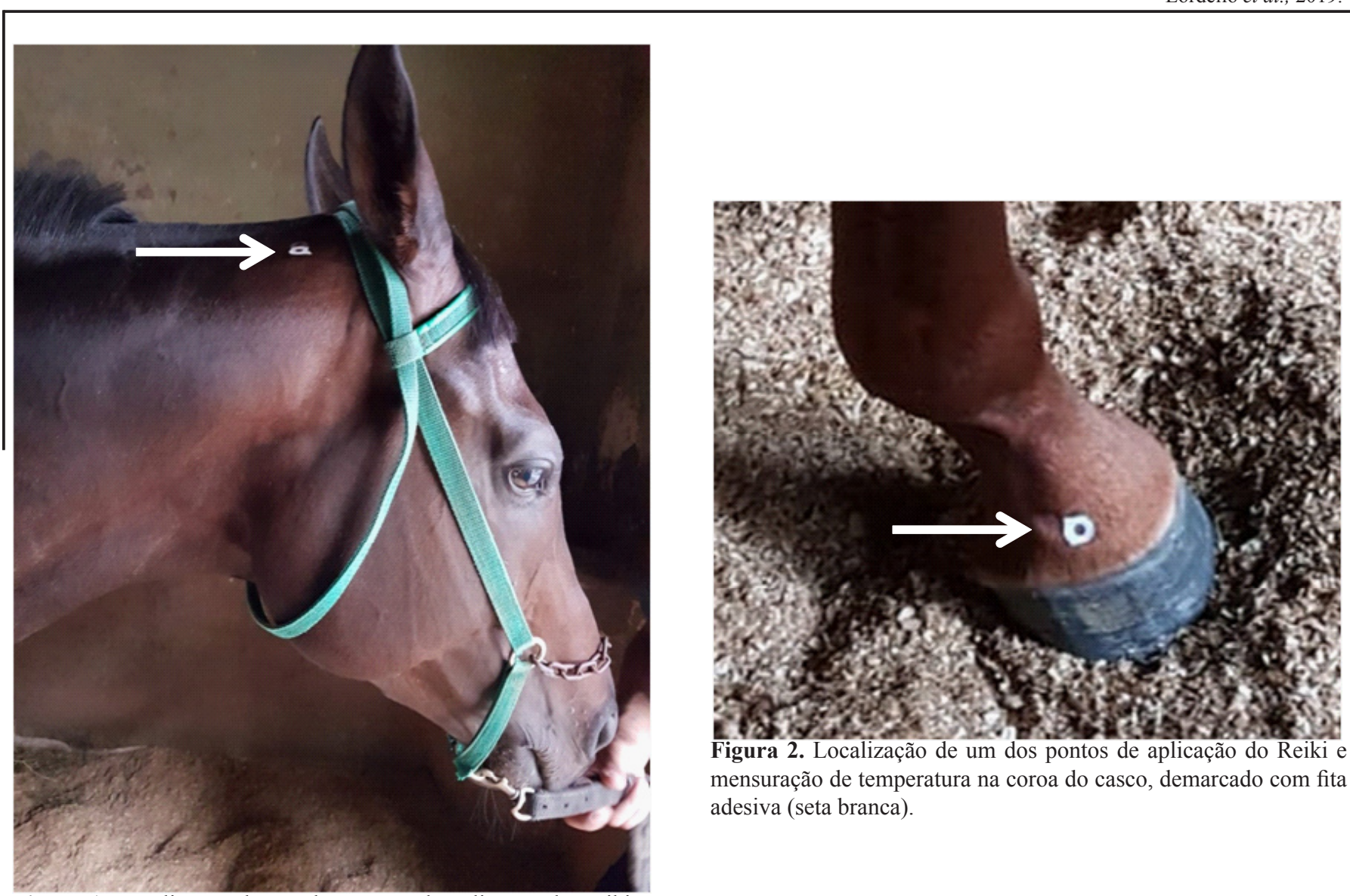

Figura 2. Localização de um dos pontos de aplicação do Reiki e mensuração de temperatura na coroa do casco, demarcado com fita adesiva (seta branca).

Figura 1. Localização de um dos pontos de aplicação do Reiki e mensuração de temperatura no corpo do cavalo, demarcado com fita adesiva (seta branca). As mensurações foram realizadas com o marcador laser do aparelho sempre centrado no círculo do adesivo de cor branca.

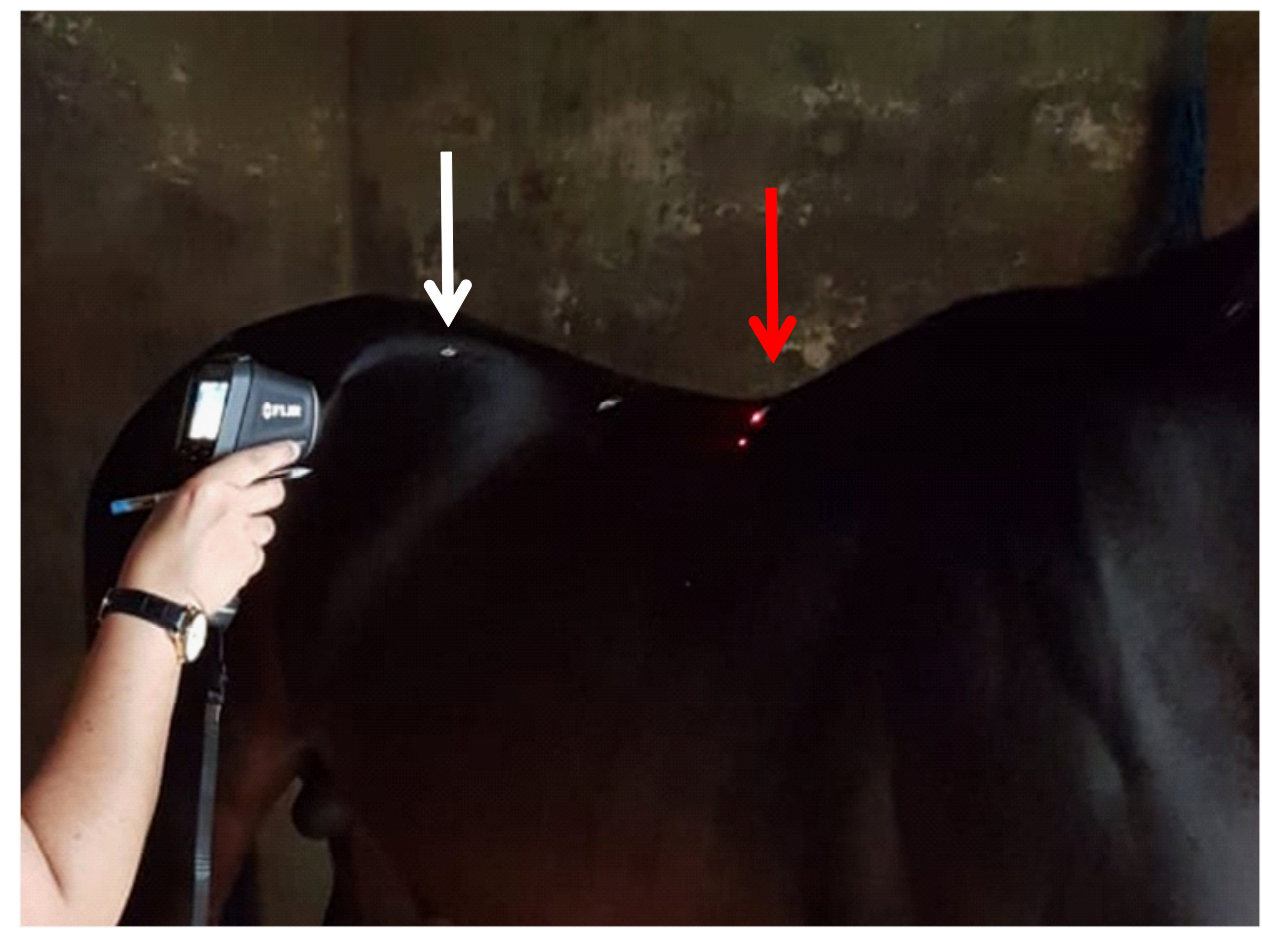

Figura 3. Mensuração em progresso e aparelho utilizado (FLIR modelo TG165, seta branca). Podese notar os dois pontos de laser no dorso do animal (seta vermelha). $O$ ponto superior foi o utilizado como referência. 
membros dos animais, conforme descrito na seção de material e métodos, foram marcados com fita adesiva como pode ser visto nas figuras acima.

As maiores médias de temperatura corporal foram observadas no ponto 2 , antes $(37,33 \pm 1,61)$ e após $(36,45 \pm 1,17)$ o tratamento, enquanto que as menores foram provenientes do ponto 1 antes $(34,92 \pm$ $1,58)$ e após $(34,08 \pm 1,38)$ o tratamento. As médias e desvio padrão das temperaturas obtidas em cada um dos 7 pontos de mensuração no corpo dos animais antes, durante (ao começo e após a primeira passagem sobre os chacras) e depois da aplicação do Reiki podem ser visualizados na tabela 1 , abaixo. Já a tabela 2 mostra as médias e os desvio padrão dos pontos localizados próximos às coroas de cada um dos cascos. A maior temperatura de superfície nos membros foi observada no anterior direito durante o tratamento $(33,07 \pm 1,02)$ e a menor foi mensurada no posterior direito, após o tratamento $(31,45 \pm 1,40)$.
Os gráficos (Figuras 4 e 5) mostram a distribuição e a variação da temperatura em cada ponto e a cada momento do experimento.

A análise de variância de dois fatores (two-way ANOVA) demonstrou que a variação de temperatura do corpo dos animais foi oriunda do ponto de mensuração $(30,65 \% ; p=0,006)$, indivíduo $(48,45 \%$, $\mathrm{p}<0,0001)$ e momento do tratamento $(1,75 \% ; \mathrm{p}=$ 0,018). Comparações múltiplas com pós teste de Dunnet utilizando a temperatura antes do tratamento como referência demonstraram que as médias das temperaturas durante o tratamento (D1 e D2) não foram significativamente diferentes da média das temperaturas iniciais ( $q=0,24$ e 0,49 , respectivamente), enquanto que a média das temperaturas ao fim do tratamento foi significativamente diferente da média da temperatura inicial $(q=2,86)$. Já em relação aos pontos localizados nos membros, a análise de variância de dois fatores ( two-way ANOVA) demonstrou que a origem da variação

Tabela 1. Média e desvio padrão (D.P.) das temperaturas de superfície obtidas em 7 pontos sobre o corpo de 6 cavalos da raça Puro-Sangue Inglês, em repouso, antes, durante (D1 e D2) e após a aplicação de tratamento de Reiki por pessoa qualificada. Legenda: Antes = antes do tratamento, D1 e D2 = tempos durante o tratamento, Após $=$ após o tratamento.

\begin{tabular}{llllllll} 
Momento & 1 & 2 & 3 & 4 & 5 & 6 & 7 \\
\hline Antes & 34,92 & 37,33 & 36,98 & 35,87 & 36,23 & 36,37 & 36,37 \\
\hline D.P. & 1,58 & 1,61 & 1,94 & 1,32 & 1,42 & 1,25 & 1,25 \\
\hline Durante 1 & 35,28 & 36,63 & 36,62 & 36,12 & 36,07 & 36,42 & 36,42 \\
\hline D.P. & 1,48 & 1,29 & 1,39 & 0,70 & 1,11 & 0,95 & 0,95 \\
\hline Durante 2 & 34,67 & 36,67 & 36,88 & 36,07 & 36,37 & 36,30 & 36,30 \\
\hline D.P. & 1,30 & 0,89 & 1,15 & 1,03 & 0,74 & 0,80 & 0,80 \\
\hline Após & 34,08 & 36,45 & 36,38 & 35,92 & 36,03 & 35,82 & 35,82 \\
\hline D.P. & 1,38 & 1,17 & 1,81 & 1,06 & 0,71 & 0,80 & 0,80
\end{tabular}

Tabela 2. Média e desvio padrão (D.P.) das temperaturas de superfície obtidas em 4 pontos nas extremidades dos membros de 6 cavalos da raça Puro-Sangue Inglês, em repouso, antes, durante (D2) e após a aplicação de tratamento de Reiki por pessoa qualificada. Legenda: Antes = antes do tratamento, Durante $=$ tempo durante o tratamento, Após = após o tratamento.

\begin{tabular}{lcccc} 
Momento & $1 \mathrm{AD}$ & $2 \mathrm{PD}$ & $3 \mathrm{AE}$ & $4 \mathrm{PE}$ \\
\hline Antes & 32,97 & 32,57 & 32,78 & 32,47 \\
\hline D.P. & 1,43 & 1,57 & 0,98 & 1,06 \\
\hline Durante & 33,07 & 32,50 & 32,12 & 31,78 \\
\hline D.P. & 1,02 & 1,98 & 2,09 & 1,69 \\
\hline Após & 32,92 & 31,45 & 32,03 & 31,55 \\
\hline D.P. & 0,74 & 1,40 & 1,21 & 1,35
\end{tabular}




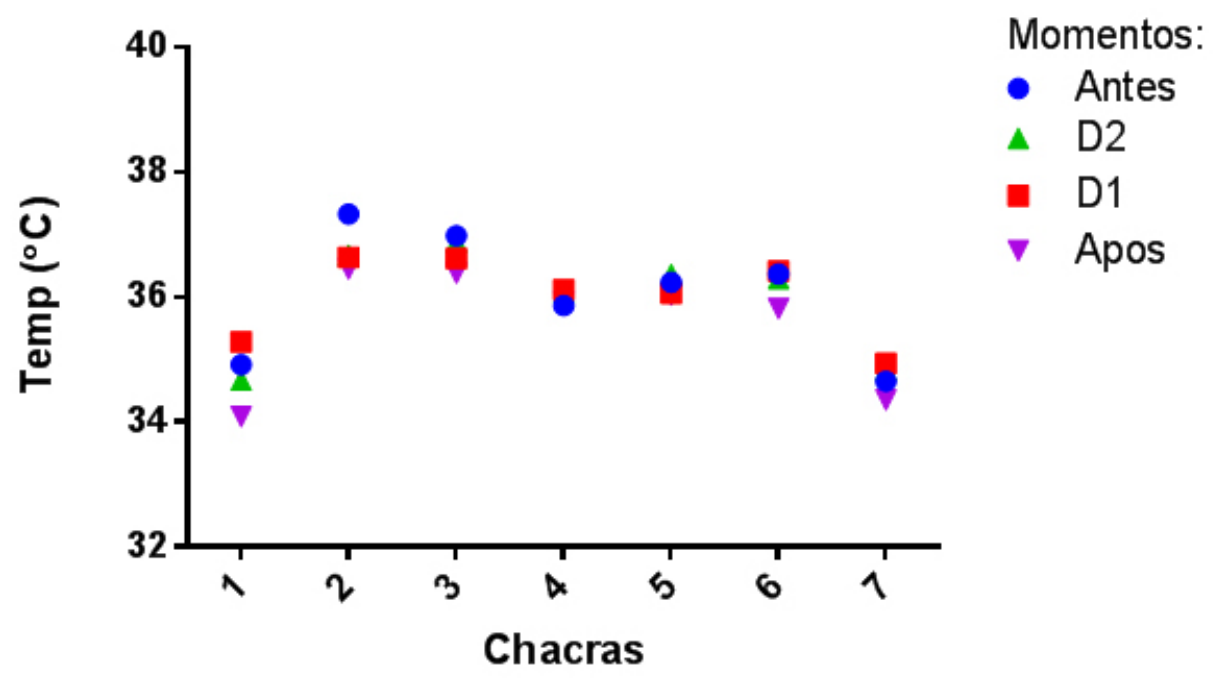

Figura 4. Gráfico demonstrando a média das temperaturas de superfície nos 7 chacras de seis equinos antes, durante (D1 e D2) e após o tratamento com Reiki por terapeuta qualificada.

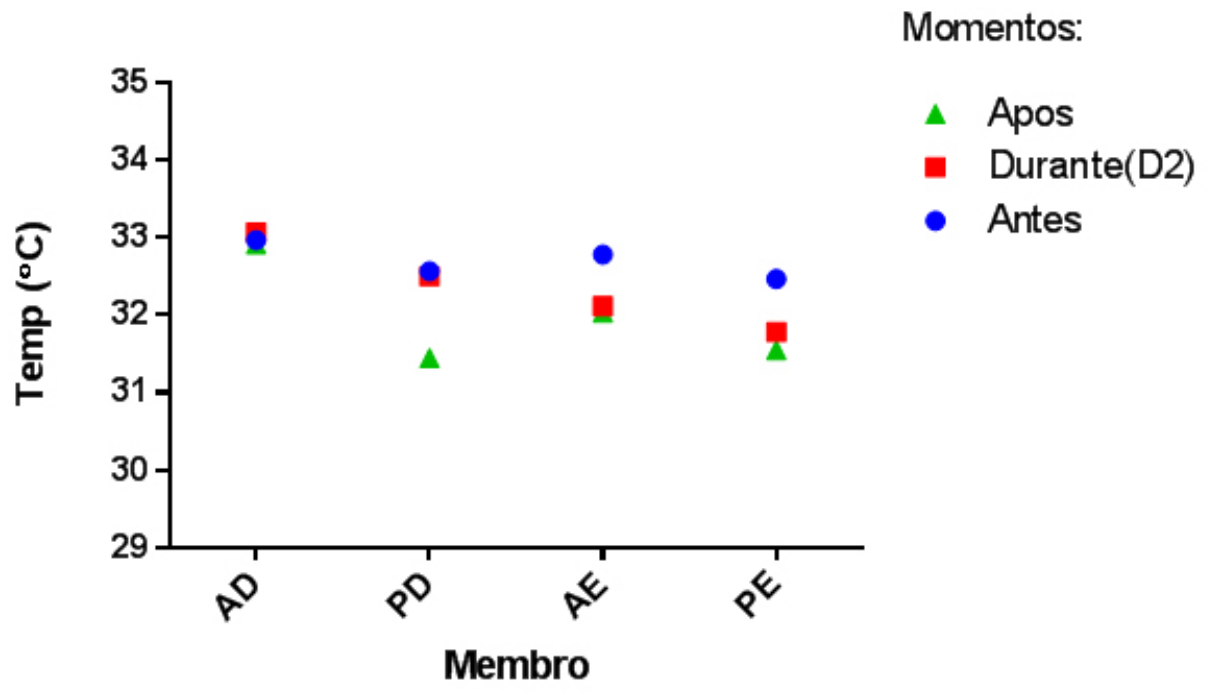

Figura 5. Gráfico demonstrando a média de variação das temperaturas de superfície nos 4 pontos dos membros de seis equinos antes, durante (D2) e após o tratamento com Reiki por terapeuta qualificada. Legenda: $\mathrm{AD}=$ anterior direito, $\mathrm{PD}=$ posterior direito, $\mathrm{AE}=$ anterior esquerdo, $\mathrm{PE}=$ posterior esquerdo.

de temperatura foi devida ao indivíduo $(62,86 \%, \mathrm{p}<$ $0,0001)$ e ao momento da aferição $(4,2 \%, p=0,034)$, não sofrendo influência do ponto de mensuração $(\mathrm{p}=$ $0,50)$.

\section{Discussão}

No caso do presente trabalho, optou-se por utilizar a coluna vertebral como ponto de localização, colocando-se os marcadores na linha média do osso frontal, acima da linha dos olhos (ponto 1, equivalente ao chacra frontal), no topo da cabeça (ponto 2, equivalente ao chacra coronário), no terço médio do pescoço (ponto
3, equivalente ao chacra laríngeo), na cernelha (ponto 4, equivalente ao chacra cardíaco), no terço médio da região lombar (ponto 5, equivalente ao chacra do plexo solar), na junção lombo-sacra (ponto 6 , equivalente ao chacra sacral) e na inserção da cauda (ponto 7 , equivalente ao chacra basal). Além disso os quatro pontos localizados nas coroas dos cascos correspondem a chacras menores, chamados de botões, do inglês $b u d^{21}$.

Apesar de haver variação de temperatura relativa ao tempo em que a mensuração foi obtida, ou seja, antes, durante e após a aplicação do Reiki, as maiores variações foram de contribuição do ponto onde a mensuração foi realizada e do indivíduo.

A média das temperaturas de superfície do corpo 
de cavalos da raça PSI variou significativamente de acordo com o ponto de mensuração, sendo mais elevadas no ponto 2 , correspondente ao chacra frontal, e mais baixas no ponto 1 , equivalente ao chacra coronário. $\mathrm{O}$ chacra frontal está localizado na região do topo da cabeça e o coronário sobre a testa ${ }^{21} \mathrm{em}$ seres humanos. Em animais, a descrição anatômica detalhada da localização dos chacras não existe na literatura científica, sendo frequentemente utilizadas transposições dos pontos descritos em humanos.

A variação de temperatura relativa ao ponto de mensuração poderia estar relacionada à quantidade de musculatura presente, sendo maior nos pontos onde houvesse mais tecido e, consequentemente maior vascularização. No entanto, a maior e a menor temperatura foram observadas em locais contíguos e com menor quantidade de tecido em comparação com outros pontos mais caudais do corpo. O presente trabalho não objetivou mensurar a quantidade de tecido ou vascularização presente em cada ponto, limitando-se à avaliação da temperatura de superfície.

Variações documentadas em medidas obtidas com aparelhos de infravermelho, como o termógrafo FLIR utilizado no presente experimento, podem ser de origem ambiental ou por falta de calibração adequada do aparelho. As diretrizes para execução de exames termográficos com equipamentos de infravermelho sugerem que deve haver um espaço de, 1 a 2 metros entre o paciente e o termógrafo, além da sala de exame estar protegida de vento e mudanças de temperaturas, devendo idealmente ser climatizada ${ }^{22}$. No caso do presente estudo, o exame foi realizado dentro de cocheiras de $3 \times 4$ metros, com amplo espaço entre o termógrafo e o animal; a mensuração ocorreu à distância calibrada de 1 metro. Apesar de não ser climatizada, a cocheira era abrigada de rajadas de vento, exposição direta à luz solar e o experimento foi realizado em dia com pouca variação térmica. Além disso, a calibração interna do aparelho permite estabilidade nas medidas obtidas pois usa uma escala interna como padrão de comparação. O uso da termografia para detecção de alterações de superfície associadas à claudicação não aparente em equinos é bastante antiga e considerada de valia como auxiliar no exame clínico ${ }^{17}$.

Um protocolo rígido de manipulação do equipamento e controle de variáveis ambientais é necessário para que comparações a longo prazo e avaliação de tratamentos farmacológicos sejam interpretados de maneira correta $^{18}$. No caso deste experimento, todas as mensurações foram realizadas no mesmo dia, sob as mesmas condições, havendo uma variação mínima do equipamento, como pode ser atestado pelo coeficiente de variação do equipamento, que foi menor do que o coeficiente de variação das medidas corporais, indicando que os resultados registrados não eram oriundos de variações no método de avaliação termográfica.

Para reduzir o efeito da temperatura ambiente sobre as medidas de temperatura de superfície, todas as coletas foram realizadas na parte da tarde, das 16 às 19. A maior variação diurna registrada no dia do experimento foi de $1,4{ }^{\circ} \mathrm{C}$, às 2 da manhã ${ }^{20}$. O dia de escolha para as mensurações foi um dia ameno, nublado mas sem chuva, contribuindo para menores extremos de temperatura ambiente, com a variação máxima registrada durante a realização do experimento de $0,5{ }^{\circ} \mathrm{C}^{20}$.

$\mathrm{O}$ aparelho foi calibrado e operado por pessoa experiente, tendo sido todas as coletas realizadas pelo mesmo pesquisador, reduzindo assim variações relacionadas ao operador.

A literatura comprova que variações de até 20 graus de angulação e de até 0,5 metros de distância não causam variações significativas na mensuração termográfica do corpo de cavalos ${ }^{19}$. Também foi demonstrado que a temperatura do corpo do animal na região dorsal à altura das vertebras torácicas e lombares tende a ser maior do que no restante do $\operatorname{corpo}^{23}$, o que não foi documentado no presente estudo. Todas as temperaturas de superfície estavam abaixo das temperaturas corporais de referência, sendo a temperatura mais alta detectada na região da cabeça. As temperaturas dos cascos estavam especialmente baixas, o que foi documentado em um estudo que detectou maior variação relativa à vasoconstricção nesta região dos equinos $^{24}$. Este achado indica ausência de inflamação local, comprovando de que, clinicamente, os animais encontravam-se livres de claudicação. A presença de inflamação aumenta significativamente a temperatura de superfície do corpo dos equinos ${ }^{18}$.

A pequena contribuição do tratamento na variação de temperatura pode ser devida ao fato de que os animais se encontravam saudáveis. Em um relato de caso anterior (Da Silva, comunicado pessoal), onde uma variação expressiva da temperatura superficial foi documentada em um cavalo, o animal encontrava-se em processo cicatricial de uma lesão contaminada e ativa, no momento da aferição e tratamento. É possível que maiores variações sejam detectáveis apenas em animais sistemicamente doentes ou com processos inflamatórios ativos. Para realizar tal investigação, grupos de animais separados por etiologia de processo poderiam ser tratados e as temperaturas superficiais avaliadas.

$\mathrm{Na}$ literatura humana há descrições bastante escassas dos efeitos da terapia Reiki, sendo possível encontrar relatos de alterações significativas em padrões hematológicos ${ }^{11}$ e na pressão arterial de pacientes tratados com Reiki ${ }^{12}$. Não foi possível encontrar um só trabalho científico publicado contendo os termos "Reiki" e "medicina veterinária" em pesquisa realizada no site PubMed e Google acadêmico, em inglês ou português. Esta falta de publicações científicas ressalta a importância de pesquisas que investiguem parâmetros 
mensuráveis em associação com o uso de tratamentos alternativos. Apesar de ser uma terapia com princípio homeostático ${ }^{7}$, com o objetivo de equilibrar o paciente, é possível que o efeito não possa ser mensurado de maneira óbvia em cavalos saudáveis.

\section{Conclusão}

A avaliação termográfica de cavalos tratados com Reiki detectou uma variação significativa de origem individual, referente ao ponto de mensuração e ao momento do tratamento. Estudos com maior número de animais e com a inclusão de medidas de temperatura corporal interna e perfil hematológico são necessários para esclarecer os efeitos mensuráveis da terapia Reiki em cavalos.

\section{Agradecimentos}

Glaucia Cristina de Oliveira, pela compra do termógrafo e ao Haras LLC pela colaboração.

\section{Referências}

1. Schoen AM. Results of a survey on educational and research programs in complementary and alternative veterinary medicine at veterinary medical schools in the United States. Journal of the American Veterinary Medical Association. 2000;216(4):502-9.

2. Memon MA, Sprunger LK. Survey of colleges and schools of veterinary medicine regarding education in complementary and alternative veterinary medicine. Journal of the American Veterinary Medical Association. 2011;239(5):619-23.

3. Araújo R, Rêgo E, Lima E, Coelho M, Vasconcelos K, Baptista R, et al. Terapia floral em gatos domésticos (Felis catus, Linnaeus, 1758) portadores do complexo da doença respiratória felina-estudo clínico e hematológico. Rev Bras P1 Med, Botucatu. 2010;12:472-81.

4. Mendonça VM, dos Santos AJ, do Nascimento IR, Oliveira MAS, de Souza Rocha S, Cabral ES. Perspectivas da fitoterapia veterinária: plantas potenciais na terapia dos animais de produção. Cadernos de Agroecologia. 2015;9(4).

5. Hare D. Complementary and alternative veterinary medicine. The Canadian Veterinary Journal. 1999;40(6):376.

6. Broadfoot PJ, Palmquist RE, Johnston K, Wen JJ, Fougere B. Integrating complementary medicine into veterinary practice: John Wiley \& Sons; 2009

7. Usui M, Petter FA. The Original Reiki Handbook of Dr. Mikao Usui: Lotus Press; 1999.

8. Miles P, True G. Reiki-review of a biofield therapy history, theory, practice, and research. Alternative Therapies in Health \& Medicine. 2003;9(2).

9. Kowalski R. The Chakras. The Mindfulness and Mindbalancing Handbook: Routledge; 2017. p. 159-76.

10. Lee MS, Pittler MH, Ernst E. Effects of reiki in clinical practice: a systematic review of randomised clinical trials. International journal of clinical practice. 2008;62(6):947-54

11. VanderVaart S, Gijsen VM, de Wildt SN, Koren G. A systematic review of the therapeutic effects of Reiki. the Journal of alternative and complementary medicine. 2009; 15(11):1157-69.
12. Mackay N, Hansen S, McFarlane O. Autonomic nervous system changes during Reiki treatment: a preliminary study. Journal of Alternative \& Complementary Medicine. 2004;10(6):1077-81

13. Olson K, Hanson J, Michaud M. A phase II trial of Reiki for the management of pain in advanced cancer patients. Journal of pain and symptom management. 2003;26(5):990-7.

14. Gronowicz GA, Jhaveri A, Clarke LW, Aronow MS, Smith TH. Therapeutic touch stimulates the proliferation of human cells in culture. The Journal of Alternative and Complementary Medicine. 2008;14(3):233-9.

15. Horowitz S. Integrative Health Care for Pets: An Overview of Today's Treatments. Alternative \& Complementary Therapies. 2003;9(6):317-21.

16. Lana SE, Kogan LR, Crump KA, Graham JT, Robinson NG. The use of complementary and alternative therapies in dogs and cats with cancer. Journal of the American Animal Hospital Association. 2006;42(5):361-5.

17. Turner TA. Thermography as an aid to the clinical lameness evaluation. Veterinary Clinics of North America: Equine Practice. 1991;7(2):311-38.

18. Soroko M, Howell K. Infrared thermography: current applications in equine medicine. Journal of Equine Veterinary Science. 2018;60:90-6. e2.

19. Westermann S, Buchner HH, Schramel JP, Tichy A, Stanek C. Effects of infrared camera angle and distance on measurement and reproducibility of thermographically determined temperatures of the distolateral aspects of the forelimbs in horses. Journal of the American Veterinary Medical Association. 2013;242(3):388-95.

20. INMET. Dados Metereológicos 2018 [Available from: http://www.inmet gov.br/portal/index.php?r=estacoes/estacoesAutomaticas.

21. Dale C. Llewellyn's Little Book of Chakras: Llewellyn Worldwide; 2017.

22. Ring E, Ammer K. Infrared thermal imaging in medicine. Physiological measurement. 2012;33(3):R33.

23. von Schweinitz DG. Thermographic diagnostics in equine back pain Veterinary Clinics of North America: Equine Practice. 1999;15(1):161-77.

24. Mogg KC, Pollitt C. Hoof and distal limb surface temperature in the normal pony under constant and changing ambient temperatures. Equine Veterinary Journal. 1992;24(2):134-9. 